\title{
A SIMPLE METHOD TO ANALYZE RADIO FREQUENCY OUTBURSTS OF QUASARS
}

\author{
H. TERÄSRANTA AND E. VALTAOJA \\ Metsähovi Radio Research Station \\ FIN-02540 Kylmälä, Finland \\ AND \\ M. LAINELA \\ Tuorla Observatory \\ FIN-21500 Piikkiö, Finland
}

Quasars have been monitored with the Metsähovi Radio Telescope since 1980 at $22 \mathrm{GHz}$ and $37 \mathrm{GHz}$. During these years we have recorded numerous outbursts in our sample. Some of the outbursts are simple in nature, while others also seem to have fine structure. Can the outbursts be modelled in a simple way?

We have taken the simplest possible assumption, starting with simple outbursts. The flux is divided into two components, the core flux, which is assumed to be constant, and the outburst flux, which is the varying component. When we subtract the core flux from the total flux, the remainder is the outburst flux. For isolated outbursts (which are rather rare) we find that both the rise and the decay of the flare are well described by the form $\ln S=a+b t$, where $S$ is the flux, $t$ the time, and $a$ and $b$ constants. In. other words, the outburst flux both rises and decays exponentially.

The flux variations of most sources look somewhat chaotic as several closely spaced flares often overlap. However, we have found out that a combination of self-similar exponential outbursts with variable amplitudes can reproduce the main features of the variations.

The model components can be used in to estimate the zero epochs and the flux density evolution (including predicted future fluxes) of VLBI components. We can also assign the right radio flare phase for correlated events in other wavebands. One example is gamma/radio correlations, where we can determine whether the events are simultaneous or time-delayed relative 

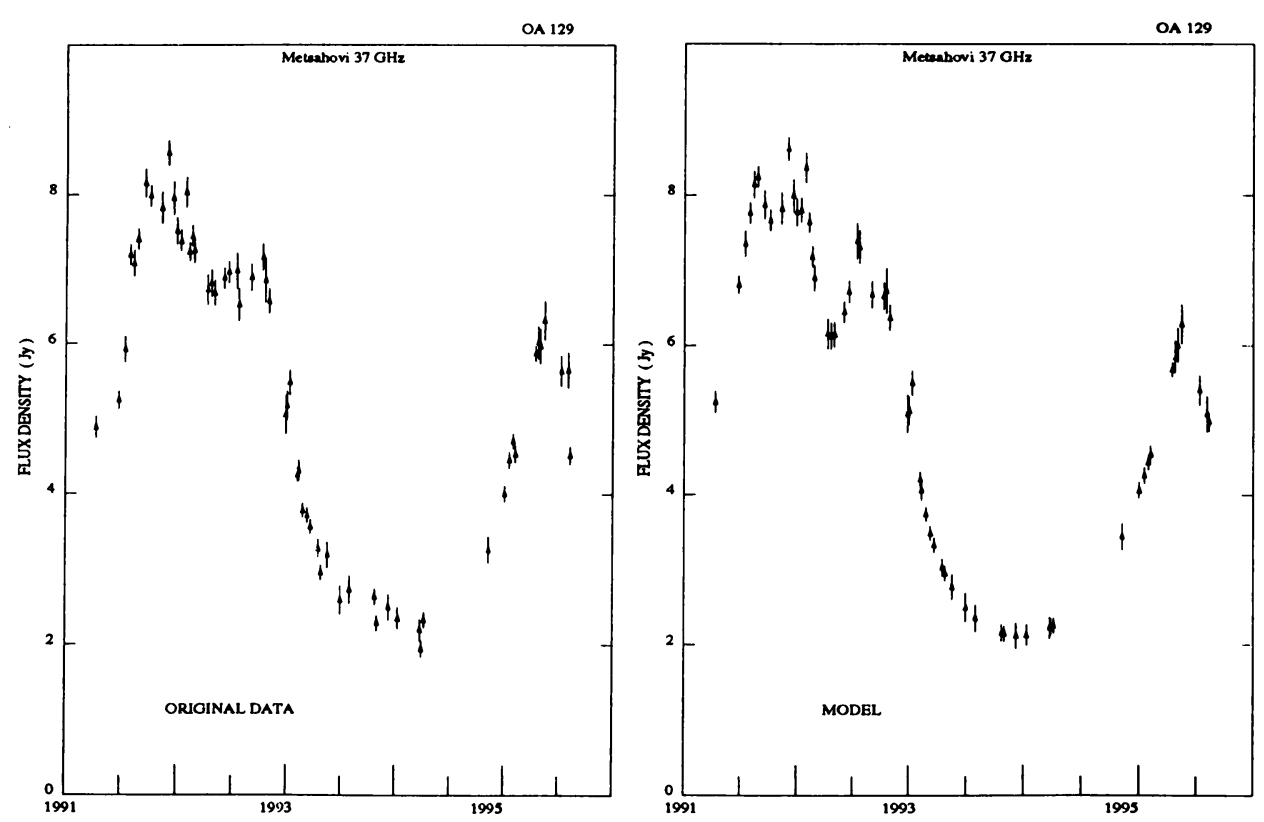

Figure 1. The time series of our $37 \mathrm{GHz}$ observations of OA 129 since 1991 and the associated model.

to each other, and if the gamma-ray emission preferentially occurs at some definite phase of the radio flare evolution.

It is remarkable that although the radio flux density variations of AGN often appear very chaotic, they can to a large extent be modelled with a small number of self-similar flares. This demonstrates that the flux variations are due to series of similar shocks in the relativistic jet, differing mainly in their strength. 